\title{
Association of early-onset diabetes, prediabetes and early glycaemic recovery with the risk of all-cause and cardiovascular mortality
}

\author{
Sung Min Kim ${ }^{1}$ - Gyeongsil Lee ${ }^{2}$ Seulggie Choi ${ }^{1}$. Kyuwoong Kim ${ }^{1} \cdot$ Su-Min Jeong ${ }^{2}$ - Joung Sik Son ${ }^{2}$. \\ Jae-Moon Yun ${ }^{2}$. Sin Gon Kim ${ }^{3}$. Seung-sik Hwang ${ }^{4}$. Seong Yong Park ${ }^{5}$ - Yeon-Yong Kim ${ }^{5}$. Sang Min Park ${ }^{1,2}$
}

Received: 15 March 2020 / Accepted: 6 July 2020 / Published online: 21 August 2020

(C) Springer-Verlag GmbH Germany, part of Springer Nature 2020

\begin{abstract}
Aims/hypothesis The increasing incidence of diabetes among young adults is a disease burden; however, the effects of earlyonset diabetes, prediabetes and glycaemic recovery on CVD or mortality remain unclear. We aimed to investigate the association of these factors with 10 year all-cause mortality, CVD mortality and CVD incidence in Korean young adults.

Methods This large and longitudinal cohort study included data from the Korean National Health Insurance Service-National Health Information Database; 2,502,375 young adults aged 20-39 years without diabetes mellitus and CVD at baseline were included. Glycaemic status was measured twice, first in 2002-2003 and second in 2004-2005. Changes in fasting glucose levels were evaluated according to fasting glucose status: normal fasting glucose (NFG; $<5.5 \mathrm{mmol} / \mathrm{l}$ ), impaired fasting glucose (IFG; 5.5-6.9 mmol/1), and diabetic fasting glucose (DFG; $\geq 7.0 \mathrm{mmol} / \mathrm{l}$ ). Primary outcomes were all-cause and CVD mortality risk. The secondary outcome was incidence of CVD, including acute myocardial infarction and stroke. All outcomes arose from the 10 year follow-up period 1 Jan 2006 to 31 December 2015.

Results Individuals with NFG at baseline, who were subsequently newly diagnosed with diabetes and prediabetes (IFG), had increased all-cause mortality (HR [95\% CI] 1.60 [1.44, 1.78] and $1.13[1.09,1.18]$, respectively) and CVD incidence (1.13 [1.05, $1.23]$ and $1.04[1.01,1.07]$, respectively). In those with DFG at baseline, early recovery to NFG and IFG was associated with decreased all-cause mortality $(0.57[0.46,0.70]$ and $0.65[0.53,0.81]$, respectively) and CVD incidence $(0.70[0.60,0.81]$ and $0.78[0.66,0.91]$, respectively). Among patients with IFG at baseline, early recovery to NFG was associated with decreased CVD mortality $(0.74[0.59,0.93])$.

Conclusions/interpretation Early-onset diabetes or prediabetes increased CVD risks and all-cause mortality after the 10 year follow-up. Furthermore, recovery of hyperglycaemia could reduce the subsequent 10 year risk for CVD incidence and all-cause mortality.
\end{abstract}

Keywords All-cause mortality $\cdot$ Cardiovascular disease $\cdot$ Diabetes $\cdot$ Glycaemic recovery $\cdot$ Prediabetes

\begin{abstract}
Abbreviations
AMI Acute myocardial infarction

CCI Charlson comorbidity index

DFG Diabetic fasting glucose
\end{abstract}

Sung Min Kim and Gyeongsil Lee are joint first authors.

Electronic supplementary material The online version of this article (https://doi.org/10.1007/s00125-020-05252-y) contains supplementary material, which is available to authorised users.

Sang Min Park

smpark.snuh@gmail.com

Extended author information available on the last page of the article
IFG
NFG
Impaired fasting glucose
NHIS National Health Insurance Service
UKPDS UK Prospective Diabetes Study

\section{Introduction}

Diabetes and its complications are significant causes of death in all countries [1]. The incidence of diabetes continues to increase worldwide despite long-term global efforts to reduce the pandemic of diabetes. The number of adults aged 


\section{Research in context}

What is already known about this subject?

- Increasing incidence of diabetes among young adults has been an additional disease burden in recent years

- Individuals with early-onset diabetes have similar or worse metabolic risk factors compared with those with lateonset diabetes

What is the key question?

- Are newly diagnosed early-onset diabetes, prediabetes and glycaemic recovery among young adults associated with incident CVD or mortality?

What are the new findings?

- In this nationwide cohort study of 2,502,375 young adults, individuals with newly diagnosed diabetes and prediabetes had increased incident CVD and all-cause mortality risk

- Individuals with early glycaemic recovery from diabetes or prediabetes had decreased incident CVD and all-cause or cardiovascular mortality risk

How might this impact on clinical practice in the foreseeable future?

- It may be particularly important to manage glycaemic levels in young adults to prevent CVD and mortality risk

$>20$ years with diabetes increased from 108 million in 1980 to 422 million in 2014 [2], and is projected to increase to 439 million by 2030 [3]. In addition to diabetes, a prediabetic status is considered to increase the risk of CVD and mortality [4]. Several meta-analyses report that hyperglycaemia is related to CVD risk in individuals without diabetes [5-7].

The increasing incidence of diabetes in young adults in recent years has become an additional disease burden [8]. According to the IDF, the diabetic population aged 20 39 years was 23 million in 2000 and steadily increased to 63 million in 2013 [9]. The disease progression of early-onset diabetes diagnosed at age 20-39 years is faster and riskier than that of late-onset diabetes diagnosed at age 40 years, likely due to longer exposure [9, 10]. Additionally, individuals with early-onset diabetes have similar or worse metabolic risk factors than those with late-onset diabetes [11].

Given these trends, early-onset prediabetes is also noteworthy; however, few studies have reported the effects of early-onset prediabetes on CVD and mortality risks. Furthermore, studies investigating whether early recovery of hyperglycaemia among young adults is effective in preventing CVD or mortality are scarce. According to the ADA and EASD, intensive behavioural lifestyle modification, including healthy eating, physical activity and smoking cessation, is the first step in preventing or delaying diabetes $[12,13]$. Intensive lifestyle modification is possible in young adults. If the effects of change in early-onset diabetes or diabetes status are related to CVD and mortality risks, their early detection and intervention are pivotal.

Therefore, this study aimed to investigate the association of early-onset diabetes, prediabetes and early glycaemic recovery with CVD risk and all-cause mortality among young adults using data obtained from the Korean National Health Insurance Service (NHIS)-National Health Information Database (NHID).

\section{Methods}

Study overview The NHIS, as the sole Korean insurer, provides mandatory health insurance for the Korean population. Among young adults aged 20-39 years, employeeinsured or self-employed workers and householders can undergo the national health screening biannually. In contrast to an earlier study, which used a $10 \%$ randomly sampled cohort [14], the data analysed in this study was obtained from a customised retrospective nationwide cohort of the NHIS database for policy research purposes and included all 20- to 39-year-old individuals who underwent health screening in 2002 and 2003 (covering insurance claims, visiting clinical facilities, diagnosis information based on International Classification of Disease [ICD] codes by the WHO, drug prescriptions) [15]. Health-screening information included sociodemographic data, physical examination results and health behaviour of enrolees.

Study population The study population was derived from NHIS database records entered between 1 January 2002 and 31 December 2015, and included all individuals aged 2039 years. Among the 2,692,643 individuals who underwent health screenings between the first (2002-2003) and second (2004-2005) periods, 1784 individuals were excluded because of missing fasting serum glucose data. Then, 
109,004 individuals who were diagnosed with type 2 diabetes before the index date (1 January 2006) based on the ICD-10 codes (tenth revision; http://apps.who.int/classifications/ icd10/browse/2016/en) for type 2 diabetes (E11, E12 and E14) and 3089 people who had taken glucose-lowering medications at least once before the index date were excluded. A further 68 people who died before the index date and 6750 who were diagnosed with CVD were excluded. A total of 69,573 individuals with missing covariate data were also excluded. Finally, 2,502,375 individuals were included in the study population (Fig. 1).

Exposures and outcomes To identify patients newly diagnosed with diabetes, prediabetes (impaired fasting glucose, IFG) and glycaemic recovery, the fasting serum glucose levels were compared between two health-screening examinations (first in 2002-2003 and second in 2004-2005) of the enrolled participants. The participants were categorised into the following three groups according to their baseline fasting serum glucose levels: normal fasting glucose (NFG) group (baseline fasting glucose level $<5.5 \mathrm{mmol} / 1[<100.0 \mathrm{mg} / \mathrm{dl}])$, IFG group (5.5$6.9 \mathrm{mmol} / 1$ [100.0-125.9 mg/dl]) and diabetic fasting glucose (DFG) group $(\geq 7.0 \mathrm{mmol} / 1[\geq 126.0 \mathrm{mg} / \mathrm{dl}])$. Among the participants placed into the NFG group at the first health examination, individuals with DFG and IFG at the second health examination were referred to as having newly diagnosed diabetes and prediabetes, respectively. Among the individuals in the DFG or IFG groups at the first health examination, those with NFG at the second health examination were regarded as exhibiting early glycaemic recovery.
The primary outcomes of the study were all-cause and CVD mortality. All-cause mortality was identified as any death between 1 January 2006 and 31 December 2015. CVD mortality was determined based on the number of CVD-related deaths between 1 January 2006 and 31 December 2015. The secondary outcomes were incident CVD, including acute myocardial infarction (AMI) and stroke. The outcomes of CVD incidence were defined as hospitalisations of at least 2 days for each disease between 1 January 2006 and 31 December 2015, based on the ICD-10 codes for these diseases (I20-I25 and I60-I69). The ICD-10 code for AMI was I21 and the codes for stroke were I60-I64 [16]. If a person was diagnosed with both AMI and stoke during the follow-up period, only the diagnosis of the first event was used [17] and the person-time was calculated based on the date of the earlier diagnosis.

Statistical analysis Cox proportional hazards regression model was used to obtain the HRs with $95 \%$ CIs for all-cause mortality, CVD mortality and CVD in the group with changed fasting serum glucose levels adjusted for age, sex, socioeconomic status, smoking status, alcohol consumption, physical activity, Charlson comorbidity index (CCI), BMI, BP and total serum cholesterol. The following covariates were obtained at the second health screening, which provided the most recent data before the index date: age; sex; socioeconomic status (quartiles, lowest to highest); BMI (<18.5, 18.5-22.9, 23-24.9 and $\geq 25 \mathrm{~kg} / \mathrm{m}^{2}$ based on the Asian obese criteria); smoking status (none, past and current); alcohol consumption as weekly drinking frequency, regardless of number of units

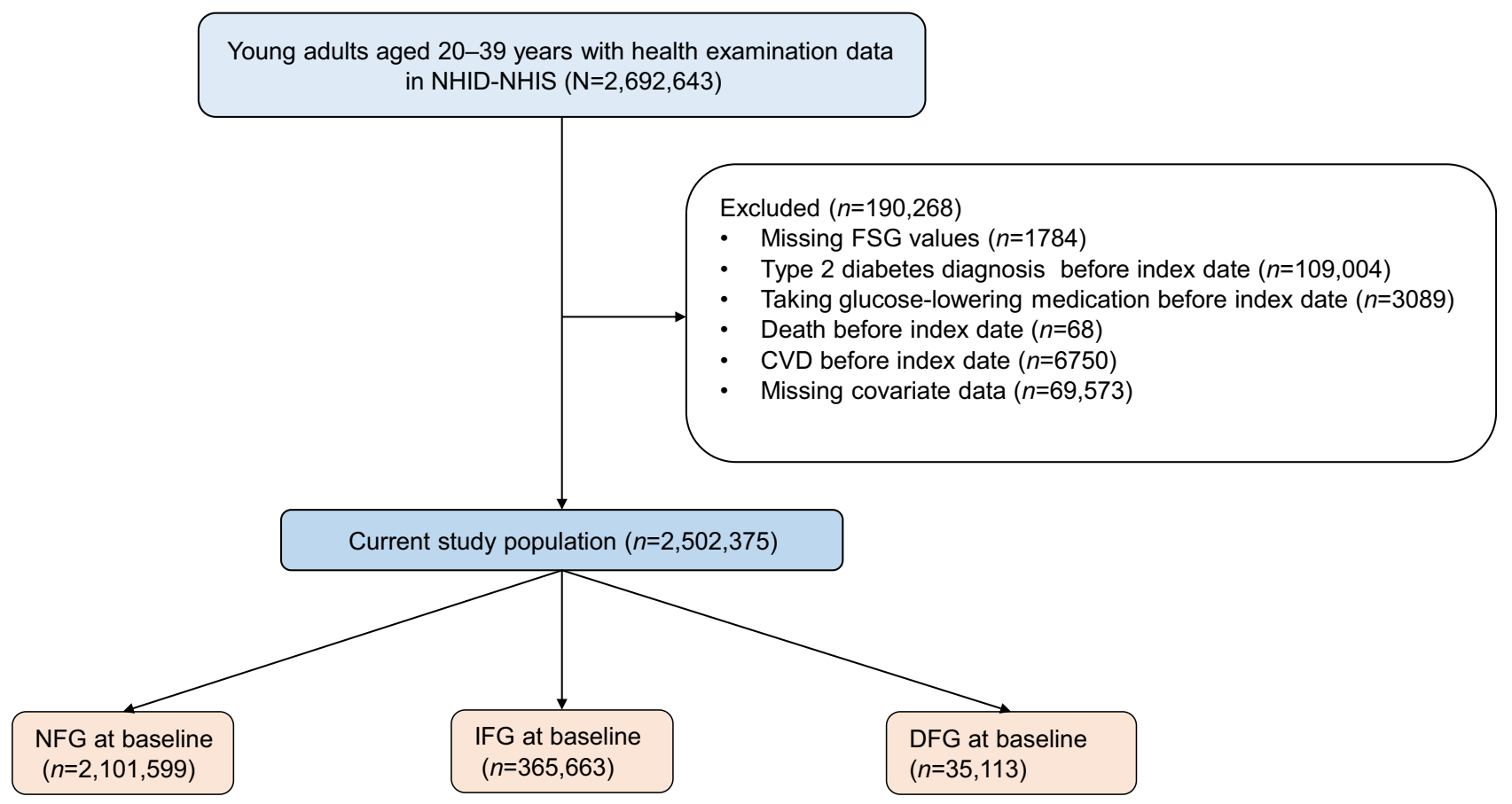

Fig. 1 Study population flow. FSG, fasting serum glucose 
consumed at a time ( $<3$ times and $\geq 3$ times per week); physical activity as weekly frequency of exercise that makes participants sweat for more than $30 \min (<3$ times and $\geq 3$ per times per week); CCI ( 0,1 and $\geq 2$ ), BP (systolic and diastolic); and total serum cholesterol level. Quartiles of insurance premium, which is based on income status, were used as a surrogate marker for socioeconomic status [15].

The groups with unchanged fasting serum glucose levels were set as the reference groups at both health examinations (i.e. NFG to NFG, IFG to IFG, and DFG to DFG). Stratified analyses were performed to identify which subgroups were significantly associated with changes in fasting serum glucose and all-cause mortality and CVD. Additionally, sensitivity analyses using multivariate-adjusted HRs were conducted, excluding those with all-cause death or CVD death or CVD in 2006 and 2007, to enhance the temporal relationship between exposure and outcome, and to exclude reversal causality. All statistical analyses were performed using SAS 9.4 (SAS Institute, Cary, NC, USA) and Stata 13.0 (StataCorp LP, College Station, TX, USA).

Ethical considerations This study was conducted according to the guidelines laid down in the Declaration of Helsinki, and all permissions regarding procedures involving human participants were waived by the Institutional Review Board of Seoul National University (IRB number: 1703-039-836). Informed consent was obtained from all participants. The NHIS database is anonymised according to strict confidentiality guidelines.

\section{Results}

The study population of 2,502,375 individuals was observed from 1 January 2006 to 31 December 2015, with a mean \pm SD follow-up of $9.9 \pm 0.4$ years resulting in 24,933,615 personyears of follow-up. The prevalence of NFG, IFG and DFG at baseline was 2,101,599 (84.0\%), 365,663 (14.6\%) and 35,113 (1.4\%), respectively. The incidence of all-cause and CVD mortality during follow-up was 21,076 (0.84\%) and 1627 $(0.07 \%)$, respectively. CVD, AMI and stroke developed in $43,877(1.75 \%), 5862(0.23 \%)$ and $12,485(0.50 \%)$ participants, respectively. Table 1 shows the general characteristics of the young adult participants classified according to their baseline fasting serum glucose levels. Participants who had a higher fasting serum glucose level were older, had lower income, had higher BMI, consumed alcohol more frequently, had higher BP and had higher cholesterol levels; a higher percentage was male and were current smokers.

The associations between early-onset diabetes, prediabetes and early glycaemic recovery and the risk of mortality are presented in Table 2. Among the participants in the NFG group at baseline, those with subsequent newly diagnosed diabetes and prediabetes had increased all-cause mortality risk compared with those in whom NFG persisted, showing a dose-response association. Among the participants in the DFG group at baseline, those who displayed early recovery from diabetes had decreased all-cause mortality risk when compared with participants in whom DFG persisted. Among participants in the IFG group at baseline, early glycaemic recovery was associated with decreased CVD mortality risk compared with persistent IFG. The associations between early-onset diabetes, prediabetes and early glycaemic recovery and CVD incidence and its subgroups (AMI and stroke) are presented in Table 3. Among participants in the NFG group at baseline, those with subsequent newly diagnosed diabetes and prediabetes had increased CVD risk compared with those in whom NFG persisted. Among participants in the IFG group at baseline, those whose fasting serum glucose levels subsequently increased to the DFG range had increased CVD risk compared with the participants in whom IFG persisted. Among participants in the DFG group at baseline, those showing early glycaemic recovery had decreased CVD risk compared with participants with persistent DFG. When comparing the changes in fasting serum glucose, the risk for CVD subgroups (AMI and stroke) showed a similar pattern to those of the main outcomes.

Subgroup analysis of the association between early-onset diabetes, prediabetes and early glycaemic recovery and the risk of all-cause mortality is shown in electronic supplementary material (ESM) Table 1. Participants who were male, aged $\geq 30$ years, with BMI $\geq 23 \mathrm{~kg} / \mathrm{m}^{2}$, and who were ever smokers had an increased risk of all-cause mortality when their fasting serum glucose level increased from the NFG range to the IFG or DFG range. Moreover, they had a decreased risk of all-cause mortality when their fasting serum glucose decreased from the DFG range to the NFG or IFG range. These participants also had an increased risk of CVD when their fasting serum glucose increased from the NFG range to the IFG or DFG range and their CVD risk decreased when their fasting serum glucose decreased from the DFG range to the NFG or IFG range (ESM Table 2). Results of the sensitivity analysis on the effect of early-onset diabetes, prediabetes and early glycaemic recovery on all-cause mortality, CVD mortality and CVD among young adults after excluding participants with all-cause mortality, CVD mortality and CVD within the first 1-2 years of follow-up are presented in ESM Tables 3, 4. These results were in line with the main findings.

\section{Discussion}

In this large and longitudinal cohort study involving more than 2.5 million young adults, we found that early-onset diabetes and prediabetes increased the CVD incidence and all-cause mortality risk after the 10 year follow-up period. 
Table 1 Baseline characteristics of study participants

\begin{tabular}{|c|c|c|c|}
\hline Characteristic & NFG & IFG & DFG \\
\hline No. of people (\%) & $2,101,599(84.0)$ & $365,663(14.6)$ & $35,113(1.4)$ \\
\hline Age, years & $33.9 \pm 5.5$ & $35.3 \pm 5.2$ & $36.0 \pm 4.9$ \\
\hline \multicolumn{4}{|l|}{ Age group, $\%$} \\
\hline $20-24$ years & 4.4 & 2.5 & 1.8 \\
\hline 25-29 years & 19.1 & 12.5 & 9.7 \\
\hline 30-34 group & 29.6 & 26.9 & 24.9 \\
\hline$\geq 35$ years & 46.9 & 58.1 & 63.6 \\
\hline \multicolumn{4}{|l|}{ Sex, $\%$} \\
\hline Men & 66.7 & 79.2 & 85.7 \\
\hline Women & 33.3 & 20.8 & 14.3 \\
\hline \multicolumn{4}{|l|}{ Socioeconomic status, $\%$} \\
\hline First level (lowest) & 11.9 & 12.1 & 13 \\
\hline Second level & 22.1 & 21.7 & 22.9 \\
\hline Third level & 36.4 & 34.9 & 35.9 \\
\hline Fourth level (highest) & 29.6 & 31.3 & 28.2 \\
\hline BMI, $\mathrm{kg} / \mathrm{m}^{2}$ & $23.0 \pm 3.2$ & $23.9 \pm 3.2$ & $24.2 \pm 3.4$ \\
\hline \multicolumn{4}{|l|}{ BMI category, $\%$} \\
\hline$<18.5 \mathrm{~kg} / \mathrm{m}^{2}$ & 5.8 & 3.5 & 3 \\
\hline $18.5-22.9 \mathrm{~kg} / \mathrm{m}^{2}$ & 46.1 & 36.9 & 33.5 \\
\hline $23-24.9 \mathrm{~kg} / \mathrm{m}^{2}$ & 22.2 & 24.5 & 24.2 \\
\hline$\geq 25 \mathrm{~kg} / \mathrm{m}^{2}$ & 25.9 & 35.1 & 39.3 \\
\hline \multicolumn{4}{|l|}{ Smoking status, $\%$} \\
\hline None & 56 & 47.3 & 39.6 \\
\hline Past & 10.4 & 12.3 & 11.9 \\
\hline Current & 33.6 & 40.4 & 48.5 \\
\hline \multicolumn{4}{|l|}{ Alcohol consumption, $\%$} \\
\hline None & 38.3 & 32.7 & 30.3 \\
\hline$<3$ times per week & 28.4 & 26.7 & 25.4 \\
\hline$\geq 3$ times per week & 33.3 & 40.6 & 44.3 \\
\hline \multicolumn{4}{|l|}{ Physical activity, \% } \\
\hline None & 51 & 47.2 & 46.2 \\
\hline$<3$ times per week & 33.2 & 35.5 & 36.9 \\
\hline$\geq 3$ times per week & 15.8 & 17.3 & 16.9 \\
\hline \multicolumn{4}{|l|}{ CCI score, $\%$} \\
\hline 0 & 52.4 & 52.3 & 54.1 \\
\hline 1 & 34.6 & 34.3 & 32.9 \\
\hline$\geq 2$ & 13 & 13.4 & 13 \\
\hline $\mathrm{sBP}, \mathrm{mmHg}$ & $118.9 \pm 13.5$ & $122.6 \pm 14.0$ & $124.8 \pm 14.9$ \\
\hline $\mathrm{dBP}, \mathrm{mmHg}$ & $75.2 \pm 9.8$ & $77.4 \pm 10.2$ & $79.1 \pm 10.6$ \\
\hline Total serum cholesterol, mmol/l & $10.3 \pm 2.1$ & $10.6 \pm 2.3$ & $10.9 \pm 2.3$ \\
\hline
\end{tabular}

Data are expressed as mean $\pm \mathrm{SD}, n(\%)$ or $\%$

Groups were defined by baseline fasting serum glucose levels as follows: NFG, $<5.5 \mathrm{mmol} / \mathrm{l}$; IFG, $5.5-6.9 \mathrm{mmol} /$ $1 ; \mathrm{DFG}, \geq 7.0 \mathrm{mmol} / 1$

$\mathrm{dBP}$, diastolic BP; sBP, systolic BP
Furthermore, early recovery of hyperglycaemia could reduce the subsequent 10 year CVD risk and all-cause mortality. To our knowledge, this is the first study to investigate the association between changes in fasting serum glucose levels and CVD risk or all-cause mortality among young adults.

Previous studies have mainly reported the effect of earlyonset vs late-onset diabetes on CVD. A 7 year prospective 
Table 2 Associations between change in fasting serum glucose and all-cause mortality and CVD mortality

\begin{tabular}{|c|c|c|c|c|}
\hline Outcome & Change in FSG & No. of events & No of person-years & aHR (95\% CI) \\
\hline \multirow[t]{9}{*}{ All-cause mortality } & $\mathrm{NFG} \rightarrow \mathrm{NFG}$ & 13,368 & $17,854,518$ & 1.00 (reference) \\
\hline & $\mathrm{NFG} \rightarrow \mathrm{IFG}$ & 2895 & $2,880,281$ & $1.13(1.09,1.18)$ \\
\hline & $\mathrm{NFG} \rightarrow \mathrm{DFG}$ & 349 & 209,016 & $1.60(1.44,1.78)$ \\
\hline & $\mathrm{IFG} \rightarrow \mathrm{NFG}$ & 2393 & $2,432,880$ & $0.96(0.90,1.03)$ \\
\hline & $\mathrm{IFG} \rightarrow \mathrm{IFG}$ & 1255 & $1,083,678$ & 1.00 (reference) \\
\hline & $\mathrm{IFG} \rightarrow \mathrm{DFG}$ & 225 & 124,268 & $1.35(1.17,1.56)$ \\
\hline & $\mathrm{DFG} \rightarrow \mathrm{NFG}$ & 255 & 194,359 & $0.57(0.46,0.70)$ \\
\hline & $\mathrm{DFG} \rightarrow \mathrm{IFG}$ & 170 & 96,638 & $0.65(0.53,0.81)$ \\
\hline & $\mathrm{DFG} \rightarrow \mathrm{DFG}$ & 166 & 57,977 & 1.00 (reference) \\
\hline \multirow[t]{9}{*}{ CVD mortality } & $\mathrm{NFG} \rightarrow \mathrm{NFG}$ & 982 & $17,854,518$ & 1.00 (reference) \\
\hline & $\mathrm{NFG} \rightarrow \mathrm{IFG}$ & 233 & $2,880,281$ & $1.05(0.91,1.21)$ \\
\hline & $\mathrm{NFG} \rightarrow \mathrm{DFG}$ & 27 & 209,016 & $1.26(0.86,1.85)$ \\
\hline & $\mathrm{IFG} \rightarrow \mathrm{NFG}$ & 180 & $2,432,880$ & $0.74(0.59,0.93)$ \\
\hline & $\mathrm{IFG} \rightarrow \mathrm{IFG}$ & 135 & $1,083,678$ & 1.00 (reference) \\
\hline & $\mathrm{IFG} \rightarrow \mathrm{DFG}$ & 20 & 124,268 & $0.97(0.61,1.56)$ \\
\hline & $\mathrm{DFG} \rightarrow \mathrm{NFG}$ & 19 & 194,359 & $0.53(0.27,1.05)$ \\
\hline & $\mathrm{DFG} \rightarrow \mathrm{IFG}$ & 14 & 96,638 & $0.60(0.30,1.24)$ \\
\hline & $\mathrm{DFG} \rightarrow \mathrm{DFG}$ & 17 & 57,977 & 1.00 (reference) \\
\hline
\end{tabular}

Groups were defined by baseline fasting serum glucose levels as follows: NFG, $<5.5 \mathrm{mmol} / \mathrm{l}$; IFG, $5.5-6.9 \mathrm{mmol} /$ $1 ; \mathrm{DFG}, \geq 7.0 \mathrm{mmol} / 1$

Change in FSG was a comparison between two health-screening examinations (first in 2002-2003 and second in 2004-2005)

aHR, HR analysed by Cox proportional hazards regression analysis adjusted for age, sex, socioeconomic status, smoking status, alcohol consumption, physical activity, CCI, BMI, BP and total serum cholesterol

FSG, fasting serum glucose study reported that early-onset diabetes led to a 30-50\% increased risk of CVD, when compared with late-onset diabetes [11]. In a large cross-sectional study, individuals with early-onset type 2 diabetes had a higher CVD risk than those with late-onset type 2 diabetes [18]. One study, comparing CVD risk in young adults with early-onset diabetes compared with those without diabetes, reported that the risk of myocardial infarction or stroke was higher in the individuals with early-onset diabetes [19].

Early-onset diabetes was also found to be associated with the risk of subsequent 10 year CVD incidence, including AMI and stroke. With regard to the association between diabetes and mortality, a modelling study found that the overall life expectancy of individuals with early-onset diabetes is reduced by 14 years in men and 16 years in women, when compared with the life expectancy of individuals without diabetes [20]. We found that early-onset diabetes was associated with CVD incidence and all-cause mortality. The association between earlyonset diabetes and CVD mortality was not significant, possibly because the population in the current study might have been too young to have experienced enough events during the 10 year follow-up. Given the legacy effect of the early modification [21], further and longer follow-up studies are needed to confirm the association between early recovery and CVD mortality.
In this study, the effects of improvement in diabetic status to NFG levels among young adults could be comparable with intensive diabetes recovery. The UK Prospective Diabetes Study (UKPDS) was the first trial showing that the recovery of hyperglycaemia is beneficial [22, 23]. By contrast, relatively recent studies investigating the effects of intensive glycaemic recovery on macrovascular complications, including Action in Diabetes and Vascular Disease: Preterax and Diamicron MR Recoveryled Evaluation (ADVANCE) [24], Action to Recovery Cardiovascular Risk in Diabetes (ACCORD) [25] and the Veterans Affairs Diabetes Trial (VADT) [26], failed to show the benefit of intensive glycaemic recovery on all-cause mortality, when compared with conventional glycaemic recovery.

Differences between the UKPDS and other trials were considered to originate from the differences in the characteristics of study participants. Participants in the UKPDS had an average age of 50 years and included those who were newly diagnosed with diabetes at the time of enrolment, whereas participants in the other three trials had an average age of 62-64 years and diabetes of approximately 10 years' duration, and $30-40 \%$ of the participants had a history of CVD. Older individuals with longer disease duration and having other comorbidities are likely to be vulnerable to hypoglycaemic events, including adverse events of 
Table 3 Associations between change in fasting serum glucose and CVD, AMI and stroke

\begin{tabular}{|c|c|c|c|c|}
\hline Outcome & Change in FSG & No. of events & No. of person-years & aHR $(95 \%$ CI) \\
\hline \multirow[t]{9}{*}{ CVD } & $\mathrm{NFG} \rightarrow \mathrm{NFG}$ & 30,200 & $17,848,558$ & 1.00 (reference) \\
\hline & $\mathrm{NFG} \rightarrow \mathrm{IFG}$ & 6352 & $2,879,048$ & $1.04(1.01,1.07)$ \\
\hline & $\mathrm{NFG} \rightarrow \mathrm{DFG}$ & 586 & 208,929 & $1.13(1.05,1.23)$ \\
\hline & $\mathrm{IFG} \rightarrow \mathrm{NFG}$ & 5076 & $2,431,990$ & $0.97(0.92,1.01)$ \\
\hline & $\mathrm{IFG} \rightarrow \mathrm{IFG}$ & 2828 & $1,083,088$ & 1.00 (reference) \\
\hline & $\mathrm{IFG} \rightarrow \mathrm{DFG}$ & 431 & 124,179 & $1.12(1.01,1.24)$ \\
\hline & $\mathrm{DFG} \rightarrow \mathrm{NFG}$ & 480 & 194,289 & $0.70(0.60,0.81)$ \\
\hline & $\mathrm{DFG} \rightarrow \mathrm{IFG}$ & 327 & 96,538 & $0.78(0.66,0.91)$ \\
\hline & $\mathrm{DFG} \rightarrow \mathrm{DFG}$ & 297 & 57,887 & 1.00 (reference) \\
\hline \multirow[t]{9}{*}{ AMI } & $\mathrm{NFG} \rightarrow \mathrm{NFG}$ & 3587 & $17,838,477$ & 1.00 (reference) \\
\hline & $\mathrm{NFG} \rightarrow \mathrm{IFG}$ & 820 & $2,876,657$ & $1.00(0.93,1.08)$ \\
\hline & $\mathrm{NFG} \rightarrow \mathrm{DFG}$ & 104 & 208,560 & $1.32(1.08,1.60)$ \\
\hline & $\mathrm{IFG} \rightarrow \mathrm{NFG}$ & 646 & $2,430,167$ & $0.88(0.78,0.99)$ \\
\hline & $\mathrm{IFG} \rightarrow \mathrm{IFG}$ & 428 & $1,081,779$ & 1.00 (reference) \\
\hline & $\mathrm{IFG} \rightarrow \mathrm{DFG}$ & 82 & 123,883 & $1.24(0.98,1.57)$ \\
\hline & $\mathrm{DFG} \rightarrow \mathrm{NFG}$ & 70 & 194,031 & $0.60(0.42,0.86)$ \\
\hline & $\mathrm{DFG} \rightarrow \mathrm{IFG}$ & 66 & 96,354 & $0.87(0.61-1.24)$ \\
\hline & $\mathrm{DFG} \rightarrow \mathrm{DFG}$ & 59 & 57,702 & 1.00 (reference) \\
\hline \multirow[t]{9}{*}{ Stroke } & $\mathrm{NFG} \rightarrow \mathrm{NFG}$ & 8009 & $17,821,125$ & 1.00 (reference) \\
\hline & $\mathrm{NFG} \rightarrow \mathrm{IFG}$ & 1750 & $2,873,326$ & $1.07(1.02,1.13)$ \\
\hline & $\mathrm{NFG} \rightarrow \mathrm{DFG}$ & 159 & 208,386 & $1.11(0.95,1.30)$ \\
\hline & $\mathrm{IFG} \rightarrow \mathrm{NFG}$ & 1309 & $2,427,664$ & $0.91(0.83,0.99)$ \\
\hline & $\mathrm{IFG} \rightarrow \mathrm{IFG}$ & 783 & $1,080,610$ & 1.00 (reference) \\
\hline & $\mathrm{IFG} \rightarrow \mathrm{DFG}$ & 132 & 123,786 & $1.21(1.01,1.45)$ \\
\hline & $\mathrm{DFG} \rightarrow \mathrm{NFG}$ & 128 & 193,824 & $0.58(0.45,0.77)$ \\
\hline & $\mathrm{DFG} \rightarrow \mathrm{IFG}$ & 113 & 96,189 & $0.81(0.62,1.06)$ \\
\hline & $\mathrm{DFG} \rightarrow \mathrm{DFG}$ & 102 & 57,608 & 1.00 (reference) \\
\hline
\end{tabular}

Groups were defined by baseline fasting serum glucose levels as follows: NFG, $<5.5 \mathrm{mmol} / 1$; IFG, $5.5-6.9 \mathrm{mmol} /$ $1 ; \mathrm{DFG}, \geq 7.0 \mathrm{mmol} / 1$

Change in FSG was a comparison between two health-screening examinations (first in 2002-2003 and second in 2004-2005)

aHR, HR analysed by Cox proportional hazards regression analysis adjusted for age, sex, socioeconomic status, smoking status, alcohol consumption, physical activity, CCI, BMI, BP and total serum cholesterol

FSG, fasting serum glucose intensive glycaemic recovery, and this could increase their mortality risk. Furthermore, the DCCT showed that intensive diabetes control has a long-term benefit in reducing CVD risk in individuals with diabetes aged approximately 27 years at baseline [27]. In the current study, glycaemic recovery decreased the CVD risk and all-cause mortality, indicating that intensive recovery is beneficial in reducing CVD and mortality risk not only in young and healthy individuals but also in individuals with earlyonset hyperglycaemia. Additionally, Lee et al investigated the effect of change in fasting serum glucose on the CVD risk among individuals aged 40 years, older than those in the current study, and found that there was no protective effect when fasting serum glucose level improved from late-onset IFG or DFG to NFG [14].
Few studies have reported the association between earlyonset prediabetes and CVD risk or mortality; however, many studies have investigated the association between late-onset prediabetes and the risks of CVD or mortality. Late-onset prediabetes is regarded as an independent risk factor for CVD and is associated with mortality [5-7]. The Emerging Risk Factors Collaboration reported fasting glucose levels exceeding $5.5 \mathrm{mmol} / \mathrm{l}$, but not $3.9-5.5 \mathrm{mmol} / \mathrm{l}$, to be associated with increased mortality risk among 820,900 individuals from 97 prospective studies [28]. We found that newly diagnosed early-onset prediabetes was also associated with CVD incidence and all-cause mortality. However, we found that recovery from prediabetes was associated with lower risks of AMI and stroke, although the clinical differences observed were small. In Korea, 
when young participants were noted as having an IFG or DFG status in the national health-screening programme, they received advice via a letter from a doctor, including suggestions for lifestyle modifications such as stopping smoking, healthy diet, moderation in drinking and elevating physical activity. Individuals with NFG at the second health examination, even though they had IFG or DFG status at the first health examination, could perform these lifestyle modifications, but not individuals with maintained or increasing hyperglycaemia.

The possible mechanism of CVD in hyperglycaemia has been reported [4, 9]. Hyperglycaemia can induce oxidative stress in the vasculature, leading to disruption of the normal endothelial function and impaired relaxation of the arterial vascular smooth muscle cells [29, 30]. Hyperglycaemia also causes overproduction of advanced glycosylation endproducts and cytokines, which leads to the activation of adhesion molecules and intima-media thickening [31]. The endothelial dysfunction and activation of adhesion in the vessels are also related to hypertension, dyslipidaemia and obesity. Individuals with early-onset diabetes have accompanying metabolic risk factors similar to, or even worse than, those of individuals with late-onset diabetes [11]. In this study, the majority of individuals with early-onset prediabetes or diabetes had greater vascular risk factors, including obesity and smoking, than young adults with NFG.

This study has several limitations. First, identifying the development of CVD by hospitalisation for 2 days or more for the relevant disease based on the ICD-10 codes may have possibly underestimated the actual number of CVD events. However, a previous study showed that identifying CVD events using the ICD-10 codes has an accuracy rate of higher than $80 \%$ [32]. On the contrary, overestimation of all-cause mortality was possible because pre-existing comorbidities may have caused relevant events in the first to the second year after the index year. However, the association between changes in fasting serum glucose level and all-cause mortality remained significant after excluding deaths from the first 2 years of follow-up. Second, a previous study has reported that impaired glucose tolerance $(2 \mathrm{~h}$ post glucose load of 7.7-10.9 mmol/l) is superior to IFG (fasting glucose level of 5.5-6.9 mmol/l) in evaluating the risk of CVD because prediabetes diagnosed by IFG is underestimated more than prediabetes diagnosed by impaired glucose tolerance [13]. However, most large cohorts have reported that prediabetes based on IFG is associated with CVD risk and all-cause mortality [28, 33, 34]. Third, the interval between baseline and glycaemic progression or regression was 2 years, which was a relatively short period. We think a longer interval between baseline and glycaemic progression or regression might alter the results; thus, further study with a longer interval is needed. Last, fasting glucose level was evaluated via serum, not plasma, as was previously recommended [35]. For fasting plasma glucose, a sodium fluoride tube must be used to inhibit glycolysis during transport to analytical centres, and another blood collection is performed, which increases the cost of the test. On the contrary, an error of $1.15 \%$ exists in the serum analyses, compared with the plasma analyses, when the sample is stored at room temperature after drawing the blood [36]. However, the error is only minimal, and the NHIS recommends refrigeration of samples at healthscreening centres. Collectively, the method used to determine the fasting serum glucose level used in the Korean NHIS has a low error rate compared with the method used for fasting plasma glucose level and applies to most people. Additionally, these findings show representative results based on data from a nationwide NHIS database [15].

In conclusion, development of early-onset prediabetes or diabetes in Korean young adults was associated with increased CVD risk and all-cause mortality after a 10 year follow-up, when compared with individuals in whom NFG or IFG persisted. Additionally, improvement of hyperglycaemia, compared with persistent hyperglycaemia, was associated with reduced CVD risk and all-cause mortality.

Acknowledgements Relating to potential overlap with a previous study [14] on a similar topic, the authors declare that the two studies differ in the data used, study design, study participants and the derived results.

Data availability Data analysed in this study are not available for public use but researchers who have a valid reason and who obtain permission from the NHIS can access it (see https://nhiss.nhis.or.kr/bd/ab/ bdaba032eng.do for details).

Funding This research was supported by a grant from the Korea Health Technology R\&D Project through the Korea Health Industry Development Institute (KHIDI), funded by the Ministry of Health \& Welfare, Republic of Korea (grant no. 20170322652-00). SMK, SC and KK received grants from the BK21-plus education programme from the National Research Foundation of Korea. This study used NHIS data (NHIS-2017-1-143) from the Korean NHIS.

Authors' relationships and activities The authors declare that there are no relationships or activities that might bias, or be perceived to bias, their work.

Contribution statement SMK, GL and SMP conceptualised and designed the study. SYP and Y-YK acquired data. SMK conducted the statistical analysis and wrote the Methods and Results sections, and GL wrote the other sections, including the Abstract, Introduction and Discussion. All authors conducted data analysis or interpretation, and critically revised the manuscript to express important intellectual content in a clearer manner. SMP had full access to all of the data in the study and took responsibility for the integrity of the data and the accuracy of the data analysis. All authors approved the final manuscript.

\section{References}

1. Smith-Spangler CM, Bhattacharya J, Goldhaber-Fiebert JD (2012) Diabetes, its treatment, and catastrophic medical spending in 35 developing countries. Diabetes Care 35(2):319-326. https://doi. org/10.2337/dc11-1770 
2. NCD Risk Factor Collaboration (2016) Worldwide trends in diabetes since 1980: a pooled analysis of 751 population-based studies with 4.4 million participants. Lancet 387(10027):1513-1530. https://doi.org/10.1016/S0140-6736(16)00618-8

3. Shaw JE, Sicree RA, Zimmet PZ (2010) Global estimates of the prevalence of diabetes for 2010 and 2030. Diabetes Res Clin Pract 87(1):4-14. https://doi.org/10.1016/j.diabres.2009.10.007

4. Buysschaert M, Medina JL, Bergman M, Shah A, Lonier J (2015) Prediabetes and associated disorders. Endocrine 48(2):371-393. https://doi.org/10.1007/s12020-014-0436-2

5. Coutinho M, Gerstein HC, Wang Y, Yusuf S (1999) The relationship between glucose and incident cardiovascular events. A metaregression analysis of published data from 20 studies of 95, 783 individuals followed for 12.4 years. Diabetes Care 22(2):233240. https://doi.org/10.2337/diacare.22.2.233

6. Huang Y, Cai X, Mai W, Li M, Hu Y (2016) Association between prediabetes and risk of cardiovascular disease and all cause mortality: systematic review and meta-analysis. BMJ 355:i5953. https:// doi.org/10.1136/bmj.i5953

7. Levitan EB, Song Y, Ford ES, Liu S (2004) Is nondiabetic hyperglycemia a risk factor for cardiovascular disease?: a meta-analysis of prospective studies. Arch Intern Med 164(19):2147-2155. https://doi.org/10.1001/archinte.164.19.2147

8. Chen L, Magliano DJ, Zimmet PZ (2012) The worldwide epidemiology of type 2 diabetes mellitus: present and future perspectives. Nat Rev Endocrinol 8(4):228. https://doi.org/10.1038/nrendo.2011. 183

9. Lascar N, Brown J, Pattison H, Barnett AH, Bailey CJ, Bellary S (2018) Type 2 diabetes in adolescents and young adults. Lancet Diabetes Endocrinol 6(1):69-80. https://doi.org/10.1016/S22138587(17)30186-9

10. Reis JP, Allen NB, Bancks MP et al (2018) Duration of diabetes and prediabetes during adulthood and subclinical atherosclerosis and cardiac dysfunction in middle age: the CARDIA Study. Diabetes Care 41(12):2579-2585

11. Chan JC, Lau ES, Luk AO et al (2014) Premature mortality and comorbidities in young-onset diabetes: a 7-year prospective analysis. Am J Med 127(7):616-624. https://doi.org/10.1016/j.amjmed. 2014.03.018

12. American Diabetes Association (2018) 4. Lifestyle management: Standards of medical care in diabetes-2018. Diabetes Care 41(Suppl 1):S38-S50. https://doi.org/10.2337/dc18-S004

13. Rydén L, Grant PJ, Anker SD et al (2013) ESC Guidelines on diabetes, pre-diabetes, and cardiovascular diseases developed in collaboration with the EASD. The task force on diabetes, pre-diabetes, and cardiovascular diseases of the European Society of Cardiology (ESC) and developed in collaboration with the European Association for the Study of Diabetes (EASD). Eur Heart J 34(39):3035-3087. https://doi.org/10.1093/eurheartj/ eht108

14. Lee G, Kim SM, Choi S et al (2018) The effect of change in fasting glucose on the risk of myocardial infarction, stroke, and all-cause mortality: a nationwide cohort study. Cardiovasc Diabetol 17(1):51

15. Cheol Seong S, Kim Y-Y, Khang Y-H et al (2017) Data resource profile: the National Health Information Database of the National Health Insurance Service in South Korea. Int J Epidemiol 46(3): 799-800. https://doi.org/10.1093/ije/dyw253

16. Kim K, Park SM, Lee K (2018) Weight gain after smoking cessation does not modify its protective effect on myocardial infarction and stroke: evidence from a cohort study of men. Eur Heart $\mathrm{J}$ 39(17):1523-1531. https://doi.org/10.1093/eurheartj/ehx761

17. Lee H, Yano Y, Cho SMJ et al (2020) Cardiovascular risk of isolated systolic or diastolic hypertension in young adults. Circulation 141 (22): 1778-1786. https://doi.org/10.1161/ CIRCULATIONAHA.119.044838
18. Huo X, Gao L, Guo L et al (2016) Risk of non-fatal cardiovascular diseases in early-onset versus late-onset type 2 diabetes in China: a cross-sectional study. Lancet Diabetes Endocrinol 4(2):115-124. https://doi.org/10.1016/S2213-8587(15)00508-2

19. Hillier TA, Pedula KL (2003) Complications in young adults with early-onset type 2 diabetes: losing the relative protection of youth. Diabetes Care 26(11):2999-3005. https://doi.org/10.2337/diacare. 26.11.2999

20. Rhodes E, Prosser L, Hoerger T, Lieu T, Ludwig D, Laffel L (2012) Estimated morbidity and mortality in adolescents and young adults diagnosed with type 2 diabetes mellitus. Diabet Med 29(4):453463. https://doi.org/10.1111/j.1464-5491.2011.03542.x

21. Chalmers J, Cooper ME (2008) UKPDS and the legacy effect. N Engl J Med 359(15):1618. https://doi.org/10.1056/NEJMe0807625

22. Holman RR, Paul SK, Bethel MA, Matthews DR, Neil HA (2008) 10-year follow-up of intensive glucose control in type 2 diabetes. $\mathrm{N}$ Engl J Med 359(15):1577-1589. https://doi.org/10.1056/ NEJMoa0806470

23. UK Prospective Diabetes Study Group (1998) Intensive bloodglucose control with sulphonylureas or insulin compared with conventional treatment and risk of complications in patients with type 2 diabetes (UKPDS 33). Lancet 352(9131):837-853. https:// doi.org/10.1016/S0140-6736(98)07019-6

24. ADVANCE Collaborative Group (2008) Intensive blood glucose control and vascular outcomes in patients with type 2 diabetes. $\mathrm{N}$ Engl J Med 358(24):2560-2572. https://doi.org/10.1056/ NEJMoa0802987

25. Action to Control Cardiovascular Risk in Diabetes Study Group (2008) Effects of intensive glucose lowering in type 2 diabetes. $\mathrm{N}$ Engl J Med 358(24):2545-2559. https://doi.org/10.1056/ NEJMoa0802743

26. Duckworth W, Abraira C, Moritz T et al (2009) Glucose control and vascular complications in veterans with type 2 diabetes. $\mathrm{N}$ Engl J Med 360(2):129-139. https://doi.org/10.1056/ NEJMoa0808431

27. Nathan DM, Cleary PA, Backlund JY et al (2005) Intensive diabetes treatment and cardiovascular disease in patients with type 1 diabetes. N Engl J Med 353(25):2643-2653. https://doi.org/10. 1056/NEJMoa052187

28. Seshasai SRK, Kaptoge S, Thompson A et al (2011) Diabetes mellitus, fasting glucose, and risk of cause-specific death. N Engl J Med 364(9):829-841

29. Khatri JJ, Johnson C, Magid R et al (2004) Vascular oxidant stress enhances progression and angiogenesis of experimental atheroma. Circulation 109(4):520-525. https://doi.org/10.1161/01.CIR. 0000109698.70638.2B

30. Piconi L, Quagliaro L, Da Ros R et al (2004) Intermittent high glucose enhances ICAM-1, VCAM-1, E-selectin and interleukin6 expression in human umbilical endothelial cells in culture: the role of poly (ADP-ribose) polymerase. J Thromb Haemost 2(8): 1453-1459. https://doi.org/10.1111/j.1538-7836.2004.00835.x

31. Thomas GN, Chook P, Qiao M et al (2004) Deleterious impact of 'high normal' glucose levels and other metabolic syndrome components on arterial endothelial function and intima-media thickness in apparently healthy Chinese subjects: the CATHAY study. Arterioscler Thromb Vasc Biol 24(4):739-743. https://doi.org/10. 1161/01.ATV.0000118015.26978.07

32. Park JK, Kim KS, Kim CB et al (2000) The accuracy of ICD codes for cerebrovascular diseases in medical insurance claims. Korean J Prev Med 33(1):76-82

33. Barr EL, Zimmet PZ, Welborn TA et al (2007) Risk of cardiovascular and all-cause mortality in individuals with diabetes mellitus, impaired fasting glucose, and impaired glucose tolerance. Circulation 116(2):151-157. https://doi.org/10.1161/ CIRCULATIONAHA.106.685628 
34. Kim H-K, Kim C-H, Kim EH et al (2013) Impaired fasting glucose and risk of cardiovascular disease in Korean men and women. Diabetes Care 36(2):328-335. https://doi.org/10.2337/dc12-0587

35. Marathe PH, Gao HX, Close KL (2017) American Diabetes Association standards of medical care in diabetes 2017. J Diabetes 9(4):320-324. https://doi.org/10.1111/1753-0407.12524
36. Frank EA, Shubha M, D'souza CJ (2012) Blood glucose determination: plasma or serum? J Clin Lab Anal 26(5):317-320. https:// doi.org/10.1002/jcla. 21524

Publisher's note Springer Nature remains neutral with regard to jurisdictional claims in published maps and institutional affiliations.

\section{Affiliations}

\section{Sung Min Kim ${ }^{1}$ - Gyeongsil Lee ${ }^{2}$. Seulggie Choi ${ }^{1} \cdot$ Kyuwoong Kim $^{1}$-Su-Min Jeong ${ }^{2}$. Joung Sik Son ${ }^{2} \cdot$ Jae-Moon Yun $^{2}$. Sin Gon Kim ${ }^{3}$ · Seung-sik Hwang ${ }^{4}$. Seong Yong Park ${ }^{5}$ Yeon-Yong Kim ${ }^{5}$. Sang Min Park ${ }^{1,2}$}

1 Department of Biomedical Sciences, Seoul National University Graduate School, Seoul, South Korea

2 Department of Family Medicine, Seoul National University Hospital, Seoul, South Korea

3 Division of Endocrinology and Metabolism, Department of Internal Medicine, College of Medicine, Korea University, Seoul, South Korea
Department of Public Health Science, Graduate School of Public Health, Seoul National University, Seoul, South Korea

5 Big Data Steering Department, National Health Insurance Service, Wonju, South Korea 